\title{
ROTUND COMPLEX NORMED LINEAR SPACES
}

\author{
P. R. BEESACK, E. HUGHES AND M. ORTEL
}

\begin{abstract}
We show that rotundity in a complex normed linear space is equivalent to the property that for any distinct vectors $x$ and $y$ of unit norm, a complex number $\alpha$ may be found for which $\|\alpha x+(1-\alpha) y\|<1$. This leads to a natural proof of a result due to Taylor and Foguel on the uniqueness of Hahn-Banach extensions.
\end{abstract}

A normed linear space $(X,\|\cdot\|)$ is rotund (or strictly convex) if whenever $x, y \in X$ with $x \neq y$ and $\|x\|=\|y\|=1$ we have $\left\|\frac{1}{2}(x+y)\right\|<1$. It is well known that in this case we actually have $\|t x+(1-t) y\|<1$ for $0<t<1$. Moreover (cf. A. E. Taylor [1, p. 544]) it is easy to see that if $X$ is not rotund there exists a real line segment $t x+(1-t) y, 0 \leqslant t \leqslant 1(x \neq y)$, all of whose points lie on the surface of the unit ball, i.e., $\|t x+(1-t) y\|=1$, $0 \leqslant t \leqslant 1$.

All of this applies whether $X$ is a real or complex normed linear space. In the case that $X$ is a complex linear space we shall prove the following alternative characterization of rotund spaces. Geometrically the characterization states that if $X$ is not rotund there exists a complex line $\alpha x+(1-$ $\alpha) y, \alpha \in C(x \neq y,\|x\|=\|y\|=1)$, all of whose points lie outside, or on the surface of, the unit ball.

TheOREM 1. A normed linear space $(X,\|\cdot\|)$ over the complex field $C$ is rotund if, and only if, $X$ has the property (L): whenever $x, y \in X$ with $x \neq y$ and $\|x\|=\|y\|=1$ we have $\|\alpha x+(1-\alpha) y\|<1$ for some $\alpha \in C$.

Proof. Clearly if $X$ is rotund then the property (L) holds with $\alpha=\frac{1}{2}$, so it is enough to prove the converse.

Suppose, then, that $X$ is a complex normed linear space. We shall prove that if $X$ is not rotund then $X$ does not have property (L). Suppose that $x, y \in X$ with $x \neq y$ and $\|x\|=\|y\|=\|(x+y) / 2\|=1$. By the HahnBanach theorem, there is an $x^{*} \in X^{*}$ such that $\left\|x^{*}\right\|=1=x^{*}((x+y) / 2)$. So $x^{*} x / 2+x^{*} y / 2=1$ and $\left|x^{*} x\right| \leqslant 1,\left|x^{*} y\right| \leqslant 1$, and since 1 is an extreme point of the unit disk it follows that $x^{*} x=x^{*} y=1$. Hence for any complex number $\alpha$,

$$
\|\alpha x+(1-\alpha) y\| \geqslant\left|x^{*}(\alpha x+(1-\alpha) y)\right|=1,
$$

and so property $(\mathrm{L})$ fails.

Received by the editors August 3, 1978.

AMS (MOS) subject classifications (1970). Primary 46B99; Secondary 46B05.

Key words and phrases. Rotund, strictly convex, Hahn-Banach extensions. 
We remark that the above proof uses the Hahn-Banach theorem, but the axiom of choice is not really required, because a simple argument shows that it is sufficient to prove Theorem 1 for spaces of two (complex) dimensions, and so only the finite-dimensional Hahn-Banach theorem is needed. Even in two dimensions it appears difficult to give a purely geometric proof.

As an application of Theorem 1 we prove the following result due to Taylor [1, Theorem 6] and Foguel [2].

THEOREM 2. Let $X$ be a complex normed linear space. Then all bounded linear functionals defined on subspaces of $X$ have unique norm-preserving linear extensions to $X$ if, and only if, the conjugate space $X^{*}$ is rotund.

Proof. Suppose $X^{*}$ is rotund but that not all Hahn-Banach extensions are unique. That is, there is a subspace $V$ of $X$ and a bounded linear functional $v^{*}$ on $V$ having distinct linear extensions $x^{*}, y^{*} \in X^{*}$ with $\left\|x^{*}\right\|=\left\|y^{*}\right\|=$ $\left\|v^{*}\right\|$. We may assume that $\left\|v^{*}\right\|=1$, clearly. Now $z^{*}=\alpha x^{*}+(1-\alpha) y^{*}$ is a linear extension of $v^{*}$ for all $\alpha \in C$. By rotundity we have

$$
\left\|\alpha x^{*}+(1-\alpha) y^{*} \alpha\right\|<1
$$

for some $\alpha \in C$. This is impossible since, for all $\alpha$,

$$
\left\|\alpha x^{*}+(1-\alpha) y^{*}\right\| \geqslant \sup _{\substack{v \in V \\\|v\|=1}}\left|v^{*}(v)\right|=1
$$

Conversely, suppose that $X$ has the unique Hahn-Banach extension property. To see that $X^{*}$ is rotund, take any $x^{*}, y^{*} \in X^{*}$ with $x^{*} \neq y^{*}$, $\left\|x^{*}\right\|=\left\|y^{*}\right\|=1$. Let

$$
V=\operatorname{ker}\left(x^{*}-y^{*}\right)=\left\{x \in X: x^{*} x=y^{*} x\right\}
$$

and let $v^{*}$ be the restriction of $x^{*}$ (or $y^{*}$ ) to $V ; v^{*}$ has a unique extension $z^{*} \in X^{*}$ with $\left\|z^{*}\right\|=\left\|v^{*}\right\|<1$. This last inequality follows from the unique-extension property and the fact that $x^{*}$ and $y^{*}$ both extend $v^{*}$. Fix any $x_{0} \in X-V$, so $x^{*} x_{0} \neq y^{*} x_{0}$. Hence the system of equations

$$
\alpha x^{*} x_{0}+\beta y^{*} x_{0}=z^{*} x_{0}, \quad \alpha+\beta=1,
$$

has a unique solution $(\alpha, \beta) \in C^{2}$, so there exists $\alpha \in C$ such that

$$
\alpha x^{*} x_{0}+(1-\alpha) y^{*} x_{0}=z^{*} x_{0}
$$

Since $X=\operatorname{Sp}\left\{x_{0}, V\right\}$ it follows that $\alpha x^{*}+(1-\alpha) y^{*}=z^{*}$, and we now have

$$
\left\|\alpha x^{*}+(1-\alpha) y^{*}\right\|=\left\|z^{*}\right\|<1
$$

so that $X^{*}$ is rotund by Theorem 1 . 


\section{REFERENCES}

1. A. E. Taylor, The extension of linear functionals, Duke Math J. 5 (1939), 538-547. MR 1, p. 58.

2. S. R. Foguel, On a theorem by A. E. Taylor, Proc. Amer. Math. Soc. 9 (1958), 325. MR 20 \#219.

Departignt of Mathimatics, Carleton University, Ottawa, Ontarto, Canada Kis 5B6 (Current address of P. R. Beesack and E. Hughes)

Current address (M. Ortel): Department of Mathematics, University of Hawaii at Manoa, Honolulu, Hawaii 96822 